\title{
Estradiol, acting through estrogen receptor alpha, restores dimethylarginine dimethylaminohydrolase activity and nitric oxide production in oxLDL-treated human arterial endothelial cells
}

\author{
Susana Novella a,b,*, Andrés Laguna-Fernández ${ }^{\mathrm{a}}$, Macarena Lázaro-Franco ${ }^{\mathrm{a}}$, Agua Sobrino ${ }^{\mathrm{a}}$, \\ Carlos Bueno-Betí ${ }^{\mathrm{a}}$, Juan J. Tarín ${ }^{\mathrm{c}}$, Elena Monsalve ${ }^{\mathrm{a}, \mathrm{b}}$, Juan Sanchís ${ }^{\mathrm{a}, \mathrm{d}}$, Carlos Hermenegildo ${ }^{\mathrm{a}, \mathrm{b}}$ \\ ${ }^{a}$ Research Foundation, Hospital Clínico of Valencia - INCLIVA, Spain \\ ${ }^{\mathrm{b}}$ Department of Physiology, University of Valencia, Spain

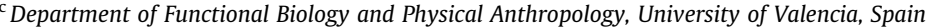 \\ ${ }^{\mathrm{d}}$ Cardiology Department, Hospital Clínico of Valencia, Spain
}

\section{A R T I C L E I N F O}

Article history:

Received 29 December 2011

Received in revised form 28 August 2012

Accepted 29 August 2012

Available online 7 September 2012

\section{Keywords:}

Asymmetric dimethylarginine

Estrogen

Nitric oxide synthase

\begin{abstract}
A B S T R A C T
Asymmetric dimethylarginine (ADMA) is an endogenous inhibitor of nitric oxide (NO) synthase. ADMA accumulation, mainly due to a decreased dimethylarginine dimethylaminohydrolase (DDAH) activity, has been related to the development of cardiovascular diseases. We investigate whether estradiol prevents the changes induced by oxidized low density lipoprotein (oxLDL) on the DDAH/ADMA/NO pathway in human umbilical artery endothelial cells (HUAEC). HUAEC were exposed to estradiol, native LDL (nLDL), oxLDL and their combinations for $24 \mathrm{~h}$. In some experiments, cells were also exposed to the unspecific estrogen receptor (ER) antagonist ICI 182780, the specific ER $\alpha$ antagonist MPP or specific agonists for ER $\alpha$, ER $\beta$ and GPER. ADMA concentration was measured by HPLC and concentration of NO by amperometry. Protein expression and DDAH activity were measured by immunoblotting and an enzymatic method, respectively. oxLDL, but not nLDL, increased ADMA concentration with a concomitant decrease on DDAH activity. oxLDL reduced eNOS protein and NO production. Estradiol alone had no effects on DDAH/ADMA/NO pathway, but increased the attenuated endothelial NO production induced by oxLDL by reduction in ADMA and preventing loss of eNOS protein levels. ICI 182780 and MPP completely abolished these effects of estradiol on oxLDL-exposed cells. ER $\alpha$ agonist, but not ER $\beta$ and GPER agonists, mirrored estradiol effects on NO production. In conclusion, estradiol restores (1) DDAH activity, and therefore ADMA levels, and (2) NO production impaired by oxLDL in HUAEC acting through ER $\alpha$.
\end{abstract}

(c) 2012 Elsevier Ireland Ltd. All rights reserved.

\section{Introduction}

Nitric oxide (NO) plays an essential role as mediator in the cardiovascular system and its release protects the vasculature against vascular diseases (Vanhoutte et al., 2009). NO is mainly produced by endothelial NO synthase (eNOS) and endothelial dysfunction is characterized by a loss of NO bioavailability. Impairment of eNOS activity by endogenous inhibitors such as asymmetrical dimethylarginine (ADMA) in endothelial dysfunction-associated diseases has gained interest (Leiper et al., 2007).

ADMA exerts its biological effects by competitively inhibiting NO synthesis and thus modulating the vascular response, and elevated levels of ADMA have been reported in many conditions associated with a high cardiovascular risk. ADMA is a metabolic

* Corresponding author at: Research Foundation - INCLIVA, Hospital Clínico Universitario of Valencia, Av. Blasco Ibañez, 17, E 46010 Valencia, Spain. Tel.: +34 96 3864900; fax: +34963864642.

E-mail address: susana.novella@uv.es (S. Novella). by-product of continuous protein turnover in cells, formed during proteolysis of methylated proteins, which is catalyzed by $S$-adenosylmethionine protein $N$-methyltransferase I (PRMT-1). Most of ADMA is metabolized to citrulline and dimethylamine through the activity of two isoforms of dimethylarginine dimethylaminohydrolase (DDAH) (Pope et al., 2009). DDAH-I is associated with tissues that express high levels of neuronal nitric oxide synthase (nNOS), whereas DDAH-II is thought to be associated with tissues that express eNOS (Leiper and Vallance, 1999). However, the biochemical properties and the contribution of each enzyme to the regulation of endothelial NO production have yet to be elucidated (Pope et al., 2009).

Oxidized low-density lipoprotein (oxLDL) acts as a risk marker for human cardiovascular disease and as an etiological factor in the pathogenesis of atherosclerosis (Steinberg, 2009). In conditions of oxidative stress, oxLDL results from oxidation of low-density lipoprotein (LDL) at the vascular wall and can cause endothelial dysfunction (Davignon and Ganz, 2004). Endothelial cells exposed to oxLDL showed many signs of dysfunction or injury, such as 
impaired NO release, disrupted endothelial barrier, decreased migration, induction of apoptosis and increased vessel stiffness which cause morphological changes (Vanhoutte et al., 2009).

The increase of ADMA level in patients and animals with hypercholesterolemia and in endothelial cells treated with oxLDL has been related to an increase of ADMA concentration as a consequence of a decreased DDAH activity, induced by oxidative stress and/or inflammatory factors (Ito et al., 1999; Boger et al., 2000).

Clinical and experimental studies have highlighted the role of estrogens in many physiological processes, among them the cardiovascular system. Indeed, experimental evidence suggest that estrogen, particularly the endogenous $17 \beta$-estradiol, exerts cardiovascular protection via the promotion of endothelial vasodilator synthesis, including NO production through both increased expression and activity of eNOS (Mendelsohn, 2009) and prostacyclin (Sobrino et al., 2010) as well as through a reduction in endothelial production of ADMA (Holden et al., 2003; Monsalve et al., 2007). These effects are mainly mediated through classical estrogen receptor (ER) $\alpha$ and ER $\beta$, which are both expressed in endothelial cells (Sobrino et al., 2010). A third type of ER, G-protein coupled which is primarily localized to the endoplasmic reticulum, named GPER, mediates several rapid and non-genomic estrogen effects (Prossnitz and Barton, 2011).

The available information on the role of ADMA in the regulation of endothelial response to estradiol has been mainly obtained in endothelial cells from venous origin. However, there is a lack of data about the effects of estradiol on DDAH/ADMA/NO pathway on arterial endothelial cells. Despite their common developmental origins, venous and arterial endothelial cells are not identical, differing widely in morphology and function. (Chi et al., 2003; Sobrino et al., 2009; dela Paz and D'Amore, 2009). Moreover, in the case of endothelial cells cultured from umbilical cord, it must take into account that in the umbilical circulation, the vein carries the oxygenated blood while the arteries carry the deoxygenated blood.

Thus, our aim was to study the interactions of a well known cardiovascular risk factor oxLDL with DDAH/ADMA/NO pathway, and the role of estradiol and ER on the observed effects in human umbilical artery endothelial cells (HUAEC).

\section{Methods}

\subsection{Cell culture and experimental design}

HUAEC were isolated from arteries from freshly human umbilical cords, as previously described (Abu-Taha et al., 2009). Briefly, both umbilical arteries were isolated, cannulated, their interior washed with PBS and then incubated 15 min with a $0.2 \%$ type II collagenase solution (Invitrogen, Barcelona, Spain). Then, the collagenase solution containing the endothelial cells was sedimented, washed twice with 199 medium (Sigma-Aldrich, Alcobendas, Spain) containing 20\% fetal bovine serum (Invitrogen). HUAEC were then grown in gelatin-coated flasks in human endothelial cell-specific medium EBM-2 (Lonza, Basel, Switzerland) supplemented with EGM-2MV (Lonza) in an incubator at $37^{\circ} \mathrm{C}$ with $5 \%$ $\mathrm{CO}_{2}$. Cells were identified as endothelial by their characteristic cobblestone morphology and the presence of von Willebrand factor by immunocytochemistry using a specific antibody (F-3520; Sigma).

Cells in confluence were exposed to $1 \mathrm{nM} 17 \beta$-estradiol (SigmaAldrich), $100 \mu \mathrm{g} / \mathrm{mL}$ native LDL (nLDL) or $100 \mu \mathrm{g} / \mathrm{mL}$ oxLDL, and their combinations. These concentrations were chosen based on our previous studies, and were able to modify DDAH/ADMA/NO pathway in other types of endothelial cells (Monsalve et al., 2007).

LDL was isolated and pooled from fasting plasma samples from healthy donors ( $\mathrm{nLDL}$ ). oxLDL was prepared by incubation with $10 \mu \mathrm{M} \mathrm{CuSO}_{4}\left(4 \mathrm{~h}\right.$ at $37^{\circ} \mathrm{C}$ ) as previously described (Monsalve et al., 2007). The effectiveness of LDL oxidation was assured by measuring malondialdehyde levels in duplicate on the nLDL as well as the oxLDL samples. Malondialdehyde levels (mean \pm SEM of 6 values corresponding to 3 experiments) were $0.7 \pm 0.1 \mathrm{nmol} / \mathrm{mg}$ protein for nLDL and $3.6 \pm 0.5$ for oxLDL $(p<0.05)$.

In some experiments, to study the role of ER, cells were also exposed to the unspecific antagonist of ER $\alpha$ and ER $\beta$ ICI 182780 $(1 \mu \mathrm{M})$, the specific ER $\alpha$ antagonist MPP $(1 \mu \mathrm{M})$, the selective ER $\alpha$ agonist PPT $(10 \mathrm{nM})$, the selective ER $\beta$ agonist DPN $(10 \mathrm{nM})$, or the selective GPER agonist G1 $(10 \mathrm{nM})$, all of them from Tocris Bioscience (Ellisville, MI, USA). Despite GPER receptors are described to mediate acute cardiovascular effects in response to estradiol (Li et al., 2012), we decided to rule out a possible interference and included the use of G1 agonist in this study.

This investigation conforms to the principles outlined in the Declaration of Helsinki, was approved by the Ethical Committee of Clinical Research of the INCLIVA, Hospital Clínico of Valencia, Spain, and written informed consent was obtained from all donors.

\subsection{Isolation and measurement of dimethylarginines}

After $24 \mathrm{~h}$ of incubation with the desired treatments, medium was stored at $-20{ }^{\circ} \mathrm{C}$ until dimethylarginines quantification and cells were collected in $0.5 \mathrm{~N} \mathrm{NaOH}$ solution for protein determination by the BCA method (Thermo Scientific Inc., Rockford, IL, USA).

Measurement of ADMA and symmetrical dimethylarginine (SDMA) was accomplished by high-performance liquid chromatography (HPLC) as described earlier (Hermenegildo et al., 2002).

\subsection{NO production}

After $24 \mathrm{~h}$ of incubation with the desired treatments, cells were washed and incubated with 5 mM HEPES containing (in $\mathrm{mM}$ ) 140 $\mathrm{NaCl}, 5 \mathrm{KCl}, 2 \mathrm{CaCl}_{2}, 1 \mathrm{MgCl}_{2}$ and 10 glucose, pH adjusted to 7.4 ), for $120 \mathrm{~min}$. Then, incubation medium was collected and stored at $-20^{\circ} \mathrm{C}$ until NO measurement. Culture wells were then washed with PBS, and adherent cells were collected in $0.5 \mathrm{~N} \mathrm{NaOH}$ solution for protein determination by the BCA method (Thermo Scientific Inc., Rockford, IL, USA). Endothelial NO production was determined using the ISONOP nitric oxide sensor (World Precision Instruments, Sarasota, FL, USA) an amperometric sensor specific for NO as described earlier (Monsalve et al., 2007).

\subsection{Immunoblotting}

Treated HUAEC were collected in RIPA buffer (Sigma) containing protease inhibitors (complete ULTRA Tablets, Roche Diagnostics, Madrid, Spain) and phosphatase inhibitors (PhosStop Tablets, Roche Diagnostics). Protein content was measured and samples were frozen at $-20^{\circ} \mathrm{C}$ until assay. Equal amounts of protein were then separated by $7-12 \%$ of SDS-Polyacrylamide gel electrophoresis. Protein was then transferred to PVDF sheets (PVDF Transfer Membrane, Bio-Rad, Madrid, Spain). Immunostaining was achieved using specific antibodies anti-PRMT-1 (sc-59647), antiDDAH-I (sc-26068), anti-DDAH-II (sc-32859), and eNOS (sc-653), all from Santa Cruz Biotechnology Inc. (Heidelberg, Germany), and anti-eNOS phosphorylated at Ser1177 (9571; Cell Signaling Technology, Danvers, MA, USA). Development was performed with horse rabbit peroxidase-linked antibodies (Sigma), followed with Supersignal Chemiluminescent Substrate (Thermo Scientific Inc.). Signal density was analyzed with ImageGauge 4.0 software. Equivalent protein loading and transfer efficiency were verified by staining for $\beta$-actin (Sigma). 


\subsection{DDAH activity assay}

A colorimetric assay was used to measure cellular DDAH activity using diacetyl monoxime derivatization of the ureido group in L-citrulline, quantified by spectrofotometry (466 nm) as previously described (Prescott and Jones, 1969).

Briefly, cells were collected by scraping in PBS $0.1 \mathrm{M} \mathrm{pH} 6.5$ and lysated in several cycles of freeze and thaw. $\mathrm{N}^{\mathrm{G}}$-monomethyl-Larginine (L-NMMA, Sigma) was added as substrate to a final concentration of $4 \mathrm{mM}$. Cell lysates were incubated at $37^{\circ} \mathrm{C}$ for $2 \mathrm{~h}$ before the addition of $30 \%$ sulfurosalicylic acid to stop the reaction. The L-citrulline formation was determined by the color development at $95{ }^{\circ} \mathrm{C}$ with an acid mixture of diacetylmonoxime $(0.8 \%$ in $5 \%$ acetic acid) and antipirine ( $0.5 \%$ in $50 \%$ sulphuric acid), and read at $466 \mathrm{~nm}$. DDAH activity was expressed as percentage of control values.

\subsection{Measurement of cell viability}

Cell viability was assessed as previously reported (Hermenegildo et al., 2005). None of the tested experimental conditions showed a percentage of viability on HUAEC lesser than $75 \%$ (data not shown).

\subsection{Statistical analysis}

Values shown in the text and figures are mean \pm Standard Error of Mean (SEM) from data obtained in 4-11 experiments. ANOVA test was applied for comparisons of mean, and then Bonferroni's test was performed. $P$ values $<0.05$ were considered significant. The statistical analysis was carried out using the Prism 4 software (GraphPad Software Inc., San Diego, CA, USA).

\section{Results}

\subsection{Dimethylarginine levels}

We first checked the effect of LDL and estradiol on dimethylarginine production by HUAEC. Exposition to oxLDL $(100 \mu \mathrm{g} / \mathrm{mL})$ for $24 \mathrm{~h}$ significantly increased the concentration of ADMA by $49 \%$ ( $p<0.05$ vs. control) in culture supernatant (Fig. 1A). By contrast, $\mathrm{nLDL}(100 \mu \mathrm{g} / \mathrm{mL})$ and estradiol $(1 \mathrm{nM})$ administered alone or in combination did not modify the concentration levels of dimethylarginines on culture supernatant. Co-exposure with oxLDL and estradiol significantly reduced the elevated concentration of ADMA (79\%; $p<0.05$ vs. control and vs. oxLDL alone), suggesting that in HUAEC estradiol only decreases ADMA levels when oxLDL is present. No changes were observed in SDMA levels after the different experimental conditions (Fig. 1B).

\subsection{NO production by HUAEC}

NO production was measured by an amperometric method based in the formation of nitrites from NO. After treatment with estradiol, nLDL and estradiol + nLDL, NO concentrations remained unchanged. Nevertheless, oxLDL $(100 \mu \mathrm{g} / \mathrm{mL})$ for $24 \mathrm{~h}$ decreased by $60 \%$ the amount of NO produced by HUAEC. Estradiol, in combination with oxLDL, stimulated NO production above control values $(124 \%)(p<0.05)$ (Fig. 2). As expected, the effects of estradiol and LDL mirrored the expected results obtained with ADMA measurement: the increased ADMA production induced by oxLDL was accompanied by a reduction in NO production, and the reduced ADMA production induced by estradiol + oxLDL was accompanied by an increase in NO production.

To study the involvement of ER, we exposed cells to ER antagonists. The increased NO production induced by estradiol + oxLDL was completely abolished by ICI 182780 , suggesting the involvement of the classic ER on that effect, and by the selective ER $\alpha$ antagonist MPP ( $p<0.05$ vs. estradiol +oxLDL), suggesting an involvement of $E R \alpha$ in the restoration of NO levels induced by estradiol in oxLDL-treated cells (Fig. 2). To further confirm that NO restoration by estradiol is only mediated by ER $\alpha$, and to discard the involvement of other ER, we studied the effect of different, specific agonists for ER $\alpha, E R \beta$ and the GPER (PPT, DPN and G1, respectively; Fig. 3). The ER $\alpha$ agonist was able to completely restore the NO levels decreased by oxLDL, to the same level than estradiol (Fig. 3), whereas neither ER $\beta$ nor GPER agonists modified the effect induced by oxLDL alone.

Taken together, the effects presented in Figs. 2 and 3 demonstrate that estradiol was able to counteract the oxLDL-reduced NO production acting through ER $\alpha$.

\subsection{Protein expression}

Our next step was to study whether these effects were due to a modification on the protein expression of the enzymes of the DDAH/ADMA/NO pathway. Western blotting analysis were performed for PRMT-1, DDAH-I, DDAH-II and eNOS proteins in HUAEC

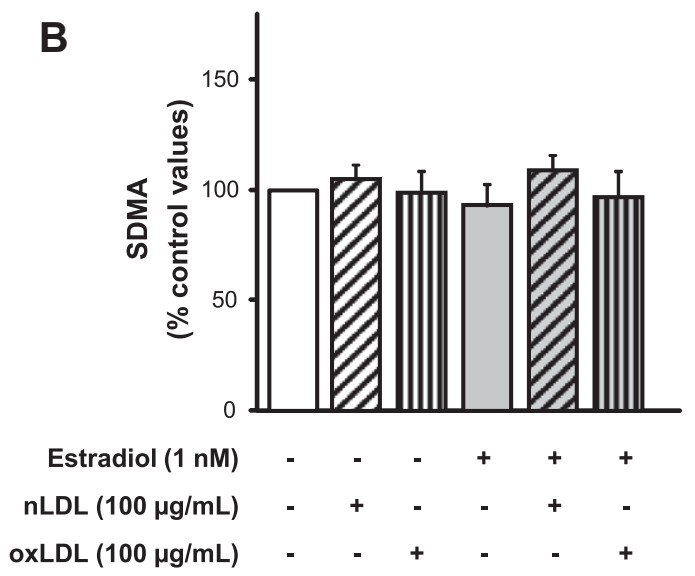

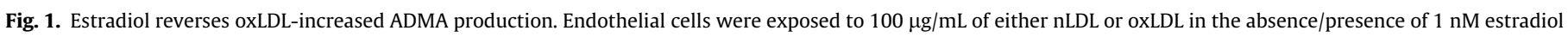

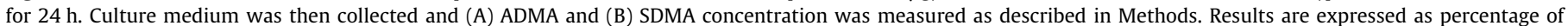

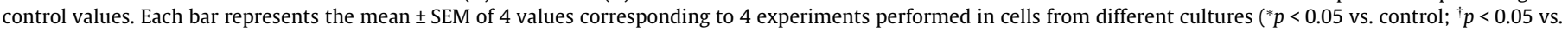
oxLDL). 


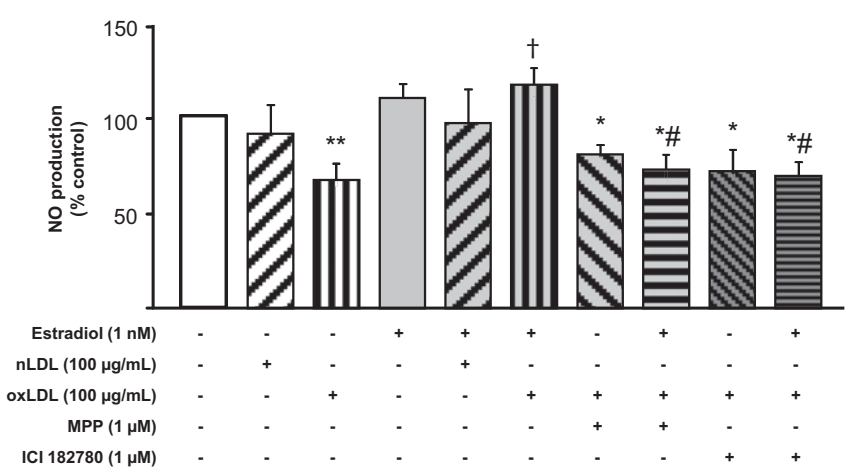

Fig. 2. oxLDL-impaired NO production is increased by estradiol through ER $\alpha$. Endothelial cells were exposed to desired treatments for $24 \mathrm{~h}$. Culture medium was then collected and NO concentration was measured as described in Methods. Results are expressed as percentage of control values and represents the mean \pm SEM of 5-12 values corresponding to 9 experiments performed in cells from different cultures $\left({ }^{*} p<0.05\right.$ vs. control; ${ }^{* *} p<0.01$ vs. control; ${ }^{\dagger} p<0.05$ vs. oxLDL; ${ }^{\#} p<0.05$ vs. oxLDL + estradiol).

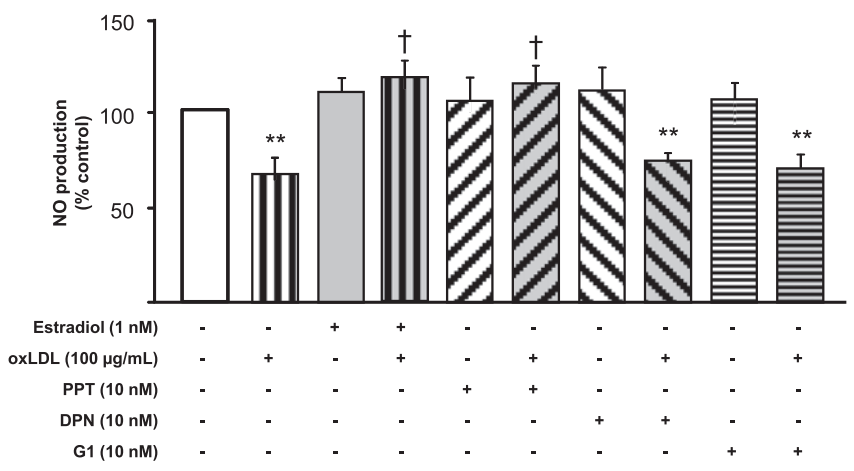

Fig. 3. ER $\alpha$ agonist selectively counteracts oxLDL-impaired NO production. Endothelial cells were exposed to desired treatments for $24 \mathrm{~h}$. Culture medium was then collected and NO concentration was measured as described in Methods. Results are expressed as percentage of control values and represents the mean \pm SEM of 5-16 values corresponding to 11 experiments performed in cells from different cultures $\left({ }^{* *} p<0.01\right.$ vs. control; ${ }^{\dagger} p<0.05$ vs. oxLDL).

exposed to estradiol, oxLDL and its combination, and $\beta$-actin was used as housekeeping protein (Fig. 4). The expression of PRMT-1 and DDAH-I were unaffected by any treatment (Fig. 4B and C). Conversely, changes in DDAH-II and eNOS expressions and eNOS phosphorylation in Ser1177 were observed after exposition to treatments (Fig. 4D-F).

DDAH-II expression remained unchanged after exposure to estradiol, was significantly increased $(p<0.05)$ in HUAEC treated with oxLDL, and remained elevated after treatment with oxLDL plus estradiol (Fig. 4D). The eNOS protein expression was significantly reduced $(p<0.05)$ by oxLDL treatment, whereas estradiol was able to completely reverse it (Fig. 4E). The activating phosphorylation of eNOS on Ser1177, as phosphorylated eNOS to total eNOS ratio, is presented in Fig. 4F. Estradiol increased eNOS phosphorylation on Ser1177/total eNOS ratio. The impaired eNOS phosphorylation after exposure to oxLDL (Fig. 4A) was related to the reduced eNOS expression (Fig. 4E), and therefore the phosphorylation ratio remained unchanged (Fig. 4F). Also, the phosphorylation ratio did not change when cells were exposed to both treatments (Fig. 4F).

\subsection{DDAH enzymatic activity}

The increased DDAH-II protein levels after exposure to oxLDL with or without estradiol did not match with ADMA levels. Therefore, DDAH activity was investigated. Neither estradiol nor nLDL modified DDAH activity, but was significantly decreased to 59\% after incubation with oxLDL $(p<0.05)$. Interestingly, estradiol not only reversed, but also stimulated DDAH activity when cells were exposed along with oxLDL $(p<0.05)$ (Fig. 5). This increment in DDAH activity (133\%) would be responsible for the low levels of ADMA observed after treatment with oxLDL plus estradiol (Fig. 1). The increased DDAH activity induced by the combination of oxLDL and estradiol was mediated through ER $\alpha$, since was completely prevented in the presence of both ICI 182780 and MPP $(p<0.05)$ (Fig. 5).

Therefore, changes observed in the DDAH activity (Fig. 5) would be the responsible for the alteration on NO production induced by oxLDL, both in the presence or in the absence of estradiol.

\section{Discussion}

Our results show how estradiol, acting through ER $\alpha$, restores DDAH activity and NO production impaired by oxLDL in HUAEC without affecting DDAH-II expression. The main findings are (1) the increment in ADMA production in arterial endothelial cells due to a diminished DDAH enzyme activity in response to oxLDL, accompanied by an increase of DDAH-II protein content, (2) the increased ADMA concentration, along with a decreased eNOS protein expression, resulted in an impaired NO production, (3) the lack of effect of estradiol on DDAH/ADMA/NO pathway on basal conditions, and (4) the restoration by estradiol of changes induced after oxLDL treatment through ER $\alpha$.

Reduced NO is a property of endothelial dysfunction. There are two important mechanisms for impaired NO synthesis, which have been studied in the present work. One of them is the alteration of eNOS phosphorylation, resulting in a modify eNOS activity, and the other one is the accumulation of endogenous NOS inhibitors such as ADMA.

On the one hand, a direct activation of eNOS by its phosphorylation on Ser1177 (Dimmeler et al., 1999) could be one of the possibilities to explain changes in NO levels. In the present study, estradiol alone do not change NO production. Although this is the first report on NO measurement under estradiol stimulation in HUAEC, in other arterial endothelial cells estradiol does not modify NO synthesis (Stewart et al., 1999).

Estradiol slightly increases eNOS phosphorylation/total eNOS ratio without changing the total eNOS expression, but this increment is not enough to induce a significant rise on NO production. A possibility to explain this discrepancy is the concomitant raise in the inhibitory phosphorylation of eNOS on Thr495 as previously described (Fleming et al., 2001). Also estradiol could be affecting other sources for NO as is the case of neuronal NOS isoform, described as a functional NO source in HUVEC (Bachetti et al., 2004).

In cells exposed to oxLDL alone, total eNOS protein was reduced, although its phosphorylation ratio remained unchanged, resulting in decreased NO. Nevertheless, when cells were exposed to estradiol + oxLDL, eNOS protein and its phosphorylation remained unchanged, in spite of in that cells NO production was significantly increased. Therefore, eNOS phosphorylation does not explain the increased NO synthesis obtained after exposure to oxLDL and estradiol.

On the other hand, in the present study oxLDL increased levels of ADMA in HUAEC. Physiological concentrations of estradiol significantly counteracted the increment in ADMA endothelial cell production induced by oxLDL concomitantly with an elevation of NO release. These results suggest that estradiol could protect cells against oxLDL-induced damage, which may involve reduction of ADMA level and increase in NO release.

The mechanisms of this action seem to differ from those previously described. Arterial endothelial cells express the enzymes re- 

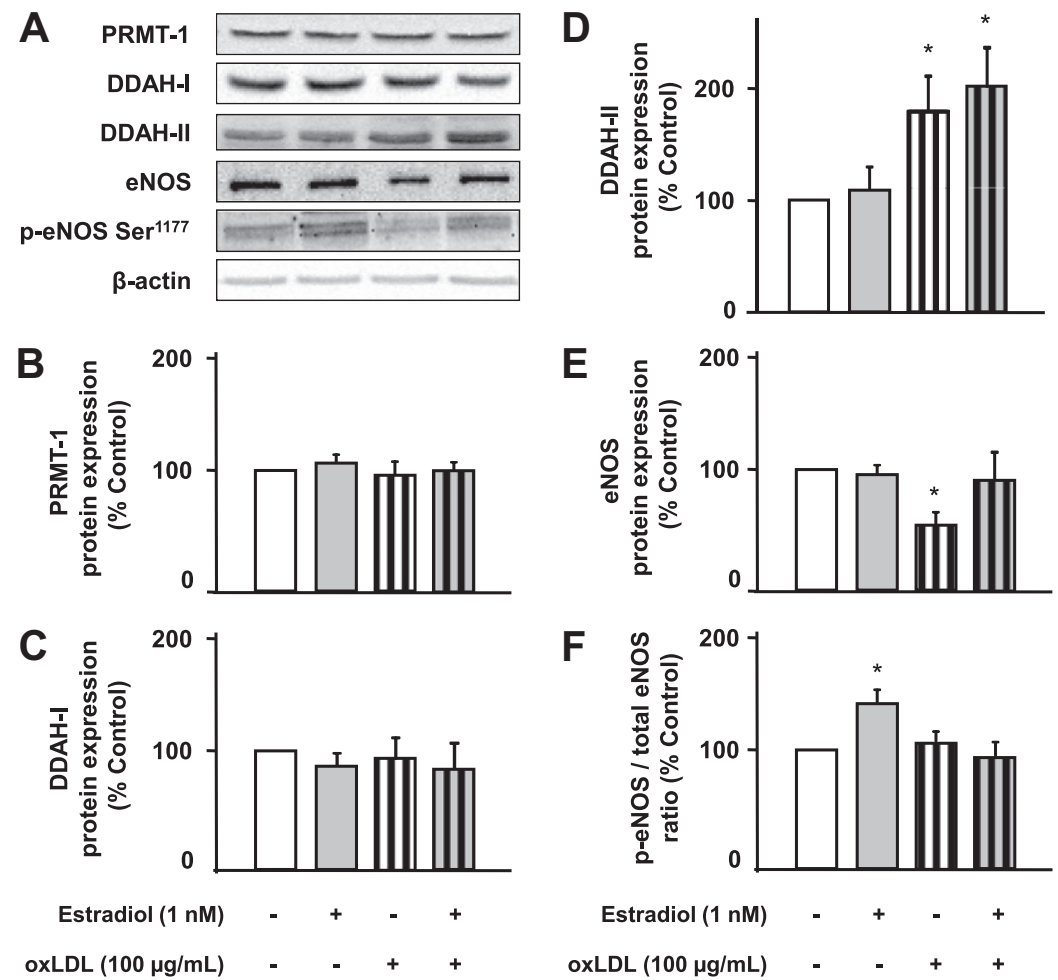

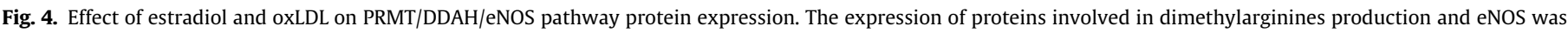

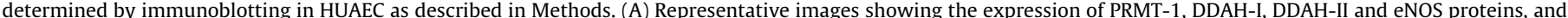

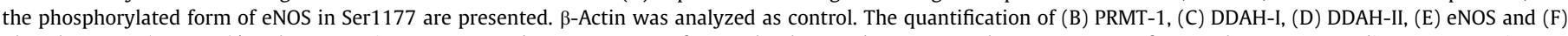

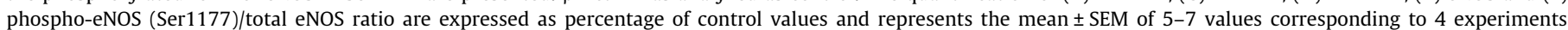
performed in cells from different cultures $\left({ }^{*} p<0.05\right.$ vs. control).

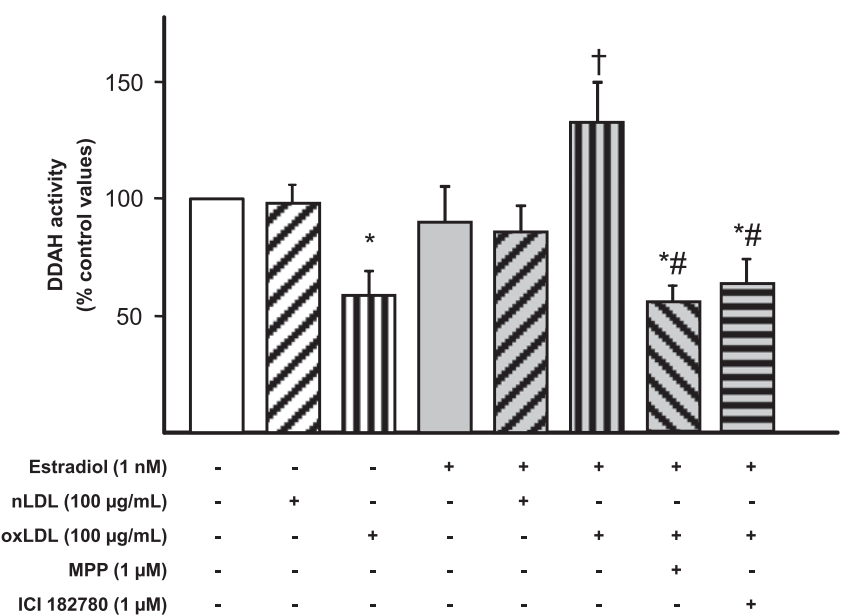

Fig. 5. oxLDL-impaired DDAH activity is increased in presence of estradiol through $\mathrm{ER} \alpha$. DDAH enzymatic activity was measured in HUAEC lysates as described in Methods. Results are expressed as percentage of control values and represents the mean \pm SEM of 5-12 values corresponding to 9 experiments performed in cells from different cultures $\left({ }^{*} p<0.05\right.$ vs. control; ${ }^{\dagger} p<0.05$ vs. oxLDL; ${ }^{*} p<0.05$ vs. oxLDL + estradiol).

quired for both synthesis (PRMT-1) and metabolism (DDAH-I and DDAH-II) of ADMA, but only DDAH-II is modified by exposure to oxLDL. Contrary to expectations, the increase in DDAH-II protein expression observed in HUAEC exposed to oxLDL came with an increment in ADMA levels. That discrepancy was explained by the decrease in DDAH activity revealed after oxLDL exposure. Only in presence of estradiol, DDAH activity was restored, leading to a decrease in ADMA concentration. DDAH-II is susceptible to be oxidized by oxLDL which make to lose its enzymatic activity. So, despite the augmentation in DDAH-II protein expression, it becomes inactive, leading to an enhanced accumulation of ADMA.

Neither DDAH-I nor PRMT-1 are involved in the response of HUAEC to oxLDL and/or estradiol. The absence of estradiol stimulation on DDAH-I protein expression is in accordance with previous studies performed in murine endothelial cells (Holden et al., 2003). In fact, the expression of DDAH-II, but not DDAH-I, protein has been shown to be transcriptionally regulated in endothelial cells (Vallance and Leiper, 2004).

The other possibility to explain the increase of ADMA concentration could be an increase in arginine methylation by PRMT-1, instead of an impaired degradation by DDAH. ADMA and SDMA share the synthesis pathway through PRMT-1 but differ in the degradation pathway, as SDMA is not a substrate for DDAH. Both the unaltered PRMT-1 expression protein profile and the unaltered SDMA levels ruled out this hypothesis. In fact, SDMA remained unaltered when cells were exposed to the different treatments, which is in complete agreement with previous studies performed in HUVEC (Boger et al., 2000; Monsalve et al., 2007).

Estradiol effects on DDAH/ADMA/NO pathway are mainly mediated through ER $\alpha$. The use of competitive antagonist of ER $\alpha$ and ER $\beta$, ICI 182780, and the selective ER $\alpha$ antagonist, MPP, completely prevented the effect of estradiol on oxLDL-reduced NO production and DDAH activity. By other side, the use of specific agonists for $\mathrm{ER} \alpha, \mathrm{ER} \beta$ and GPER clearly suggest that ER $\alpha$ activation reverses the oxLDL-impaired NO production. Taken together, these results demonstrate a selective involvement of $E R \alpha$ in the reversal effect of estradiol to the oxLDL-induced changes in DDAH/ADMA/NO pathway in HUAEC. These results reinforce the central role of ER $\alpha$ on the beneficial effects exerted by estrogens on the endothelial physiology (Sobrino et al., 2010; Chalopin et al., 2010). The absence of GPER agonist effects on genomic actions is in accordance to previous reports in which GPER receptors are described 
to mediate acute cardiovascular effects in response to estradiol ( $\mathrm{Li}$ et al., 2012).

These effects of estradiol in HUAEC were different from that previously published in HUVEC (Holden et al., 2003; Monsalve et al., 2007; Sobrino et al., 2009). In spite of HUVEC are often used to study endothelial mechanisms, experiments were developed in HUAEC in an attempt to determine if those genetic differences could alter the response to estradiol and to atherogenic compounds like oxLDL. Despite their common developmental origins, endothelial cells are not identical, differing widely in morphology and function, and existing different genes expressed specifically by the endothelium of arteries (Chi et al., 2003). Fetal circulation is rather different from adult as gas exchange does not occur in the lungs but in the placenta. In spite of umbilical arteries carry deoxygenated blood at relatively low blood pressure, some evidences have shown that the specification of arterial and venous identity is largely genetically determined (dela Paz and D'Amore, 2009). In fact, phylogenetic approaches show the identity of umbilical arteries to different arteries in the body and the same occurs with veins (Chi et al., 2003).

Estradiol has also been related to ADMA levels in women. For instance, plasmatic ADMA concentrations are lower before than after menopause, suggesting an effect of estrogens in ADMA metabolism (Schulze et al., 2005). Indeed, in some studies, hormone replacement therapy significantly reduced plasma concentrations of the cardiovascular risk factor ADMA in healthy postmenopausal women (Post et al., 2003) and raised levels of NO (Saitta et al., 2001) which could lead to an increase in endothelium-dependent flow-mediated dilation observed during oral hormone replacement therapy (Vehkavaara et al., 2000).

\section{Conclusions}

Estradiol restores the DDAH enzymatic activity affected by oxLDL through ER $\alpha$, without affecting the expression of proteins involved in ADMA metabolism, DDAH-I, DDAH-II and PRMT-1. This effect decreases the accumulation of ADMA and restores NO production. The results differ from those registered in HUVEC (Monsalve et al., 2007; Peng et al., 2011) indicating differences in regulation of protein expression by oxLDL and estradiol between arterial and venous endothelial cells.

\section{Acknowledgements}

Authors are indebted to Natalia Martínez-Gil BSc. for her excellent technical assistance.

Supported by the Spanish Ministerio de Ciencia e Innovación, Instituto de Salud Carlos III - FEDER-ERDF (Grants FIS PI10/ 00518 and Red HERACLES RD06/0009/0005), Consellería de Educación, Generalitat Valenciana (Grant ACOMP/2012/218).

\section{References}

Abu-Taha, M., Rius, C., Hermenegildo, C., Noguera, I., Cerda-Nicolas, J.M., Issekutz, A.C., Jose, P.J., Cortijo, J., Morcillo, E.J., Sanz, M.J., 2009. Menopause and ovariectomy cause a low grade of systemic inflammation that may be prevented by chronic treatment with low doses of estrogen or losartan. J. Immunol. 183, 1393-1402.

Bachetti, T., Comini, L., Curello, S., Bastianon, D., Palmieri, M., Bresciani, G., Callea, F. Ferrari, R., 2004. Co-expression and modulation of neuronal and endothelial nitric oxide synthase in human endothelial cells. J. Mol. Cell. Cardiol. 37, 939945

Boger, R.H., Sydow, K., Borlak, J., Thum, T., Lenzen, H., Schubert, B., Tsikas, D., BodeBoger, S.M., 2000. LDL cholesterol upregulates synthesis of asymmetrical dimethylarginine in human endothelial cells: involvement of $S$ adenosylmethionine-dependent methyltransferases. Circ. Res. 87, 99-105.

Chalopin, M., Tesse, A., Martínez, M.C., Rognan, D., Arnal, J.F., Andriantsitohaina, R., 2010. Estrogen receptor alpha as a key target of red wine polyphenols action on the endothelium. PLoS ONE 5, e8554.
Chi, J.T., Chang, H.Y., Haraldsen, G., Jahnsen, F.L., Troyanskaya, O.G., Chang, D.S., Wang, Z., Rockson, S.G., van de Rijn, M., Botstein, D., Brown, P.O., 2003. Endothelial cell diversity revealed by global expression profiling. PNAS 100, 10623-10628.

Davignon, J., Ganz, P., 2004. Role of endothelial dysfunction in atherosclerosis. Circulation 109, III27-III32.

dela Paz, N., D’Amore, P., 2009. Arterial versus venous endothelial cells. Cell Tissue Res. 335, 5-16.

Dimmeler, S., Fleming, I., Fisslthaler, B., Hermann, C., Busse, R., Zeiher, A.M., 1999 Activation of nitric oxide synthase in endothelial cells by Akt-dependent phosphorylation. Nature 399, 601-605.

Fleming, I., Fisslthaler, B., Dimmeler, S., Kemp, B.E., Busse, R., 2001. Phosphorylation of Thr495 regulates $\mathrm{Ca}^{2+} /$ calmodulin-dependent endothelial nitric oxide synthase activity. Circ. Res. 88, e68-e75.

Hermenegildo, C., Medina, P., Peiro, M., Segarra, G., Vila, J.M., Ortega, J., Lluch, S., 2002. Plasma concentration of asymmetric dimethylarginine, an endogenous inhibitor of nitric oxide synthase, is elevated in hyperthyroid patients. J. Clin. Endocrinol. Metab. 87, 5636-5640.

Hermenegildo, C., Oviedo, P. Garcia-Perez, M.A., Tarin, J.J., Cano, A., 2005. Effects of phytoestrogens genistein and daidzein on prostacyclin production by human endothelial cells. J. Pharmacol. Exp. Ther. 315, 722-728.

Holden, D.P., Cartwright, J.E., Nussey, S.S., Whitley, G.S., 2003. Estrogen stimulates dimethylarginine dimethylaminohydrolase activity and the metabolism of asymmetric dimethylarginine. Circulation 108, 1575-1580.

Ito, A., Tsao, P.S., Adimoolam, S., Kimoto, M., Ogawa, T., Cooke, J.P., 1999. Novel mechanism for endothelial dysfunction: dysregulation of dimethylarginine dimethylaminohydrolase. Circulation 99, 3092-3095.

Leiper, J., Nandi, M., Torondel, B., Murray-Rust, J., Malaki, M., O’Hara, B., Rossiter, S., Anthony, S., Madhani, M., Selwood, D., Smith, C., Wojciak-Stothard, B., Rudiger A., Stidwill, R., McDonald, N.Q., Vallance, P., 2007. Disruption of methylarginine metabolism impairs vascular homeostasis. Nat. Med. 13, 198-203.

Leiper, J., Vallance, P., 1999. Biological significance of endogenous methylarginines that inhibit nitric oxide synthases. Cardiovasc. Res. 43, 542-548.

Li, Z.l., Liu, J.c., Liu, S.b., Li, X.q., Yi, D.h., Zhao, M.g., 2012. Improvement of vascular function by acute and chronic treatment with the GPR30 agonist G1 in experimental diabetes mellitus. PLoS ONE 7, e38787.

Mendelsohn, M.E., 2009. Estrogen actions in the cardiovascular system. Climacteric 12 (Suppl. 1), 18-21.

Monsalve, E., Oviedo, P.J., Garcia-Perez, M.A., Tarin, J.J., Cano, A., Hermenegildo, C., 2007. Estradiol counteracts oxidized LDL-induced asymmetric dimethylarginine production by cultured human endothelial cells. Cardiovasc. Res. 73, 66-72.

Peng, Z.y., Zhao, S.P., He, B.m., Peng, D.q., Hu, M., 2011. Protective effect of HDL on endothelial NO production: the role of DDAH/ADMA pathway. Mol. Cell. Biochem. 351, 243-249.

Pope, A.J., Karuppiah, K., Cardounel, A.J., 2009. Role of the PRMT-DDAH-ADMA axis in the regulation of endothelial nitric oxide production. Pharmacol Res. 60, 461-465.

Post, M.S., Verhoeven, M.O., van der Mooren, M.J., Kenemans, P., Stehouwer, C.D.A. Teerlink, T., 2003. Effect of hormone replacement therapy on plasma levels of the cardiovascular risk factor asymmetric dimethylarginine: a randomized, placebo-controlled 12-week study in healthy early postmenopausal women. J. Clin. Endocrinol. Metab. 88, 4221-4226.

Prescott, L.M., Jones, M.E., 1969. Modified methods for the determination of carbamyl aspartate. Anal. Biochem. 32, 408-419.

Prossnitz, E.R., Barton, M., 2011. The G-protein-coupled estrogen receptor GPER in health and disease. Nat. Rev. Endocrinol. 7, 715-726.

Saitta, A., Altavilla, D., Cucinotta, D., Morabito, N., Frisina, N., Corrado, F., D’Anna, R., Lasco, A., Squadrito, G., Gaudio, A., Cancellieri, F., Arcoraci, V., Squadrito, F., 2001. Randomized, double-blind, placebo-controlled study on effects of raloxifene and hormone replacement therapy on plasma no concentrations, endothelin-1 levels, and endothelium-dependent vasodilation in postmenopausal women. Arterioscler. Thromb. Vasc. Biol. 21, 1512-1519.

Schulze, F., Maas, R., Freese, R., Schwedhelm, E., Silberhorn, E., Boger, R.H., 2005 Determination of a reference value for NG, NG-dimethyl-L-arginine in 500 subjects. Eur. J. Clin. Invest 35, 622-626.

Sobrino, A., Mata, M., Laguna-Fernandez, A., Novella, S., Oviedo, P.J., Garcia-Perez, M.A., Tarin, J.J., Cano, A., Hermenegildo, C., 2009. Estradiol stimulates vasodilatory and metabolic pathways in cultured human endothelial cells. PLoS ONE 4, e8242.

Sobrino, A., Oviedo, P.J., Novella, S., Laguna-Fernandez, A., Bueno, C., Garcia-Perez, M.A., Tarin, J.J., Cano, A., Hermenegildo, C., 2010. Estradiol selectively stimulates endothelial prostacyclin production through estrogen receptor a. J. Mol. Endocrinol. 44, 237-246.

Steinberg, D., 2009. The LDL modification hypothesis of atherogenesis: an update. J. Lipid. Res. 50, S376-S381.

Stewart, K.G., Zhang, Y., Davidge, S.T., 1999. Estrogen decreases prostaglandin H synthase products from endothelial cells. J. Soc. Gynecol. Investig. 6, 322-327.

Vallance, P., Leiper, J., 2004. Cardiovascular biology of the asymmetric dimethylarginine: dimethylarginine dimethylaminohydrolase pathway. Arterioscler. Thromb. Vasc. Biol. 24, 1023-1030.

Vanhoutte, P.M., Shimokawa, H., Tang, E.H., Feletou, M., 2009. Endothelial dysfunction and vascular disease. Acta Physiol. (Oxf) 196, 193-222.

Vehkavaara, S., Hakala-Ala-Pietilä, T., Virkamäki, A., Bergholm, R., Ehnholm, C., Hovatta, O., Taskinen, M.R., Yki-Järvinen, H., 2000. Differential effects of oral and transdermal estrogen replacement therapy on endothelial function in postmenopausal women. Circulation 102, 2687-2693. 